\title{
Arginine methylation of the $B$ cell antigen receptor promotes differentiation
}

\author{
Simona Infantino, ${ }^{1,3}$ Beate Benz,,3 Tanja Waldmann, ${ }^{3}$ Manfred Jung, ${ }^{2}$ \\ Robert Schneider, ${ }^{3}$ and Michael Reth ${ }^{1,3}$
}

\begin{abstract}
${ }^{1}$ Center for Biological Signaling Studies, Faculty of Biology and ${ }^{2}$ Institute of Pharmaceutical Sciences, Albert-Ludwigs-University of Freiburg, 79104 Freiburg, Germany

${ }^{3}$ Max-Planck-Institute for Immunobiology, 79108 Freiburg, Germany
\end{abstract}

Signals processed through the $B$ cell antigen receptor (BCR) control both the proliferation and differentiation of $B$ lymphocytes. How these different signaling modes are established at the BCR is poorly understood. We show that a conserved arginine in the tail sequence of the $\lg \alpha$ subunit of the BCR is methylated by the protein arginine methyltransferase 1 . This modification negatively regulates the calcium and PI-3 kinase pathways of the BCR while promoting signals leading to B cell differentiation. Thus, Ig $\alpha$ arginine methylation can play an important role in specifying the outcome of BCR signaling.

\begin{abstract}
CORRESPONDENCE
Michael Reth:

michael.reth@

bioss.uni-freiburg.de

Abbreviations used: GST, glutathione S-transferase; HA, hemagglutinin; ITAM, immunoreceptor tyrosine-based activation motif; NIP, 4-hydroxy5-iodo-3-nitrophenyl acetyl; PI3K, PI-3 kinase; PLA, proximity ligation assay; PRMT, protein arginine methyltransferase; PTK protein tyrosine kinase; siRNA, small interfering RNA.
\end{abstract}

The BCR is composed of the membranebound $\operatorname{Ig}$ molecule and the $\operatorname{Ig} \alpha / \operatorname{Ig} \beta$ heterodimer signaling subunits. The amino acid sequence of the cytosolic tail of Ig $\alpha$ is highly conserved and contains an immunoreceptor tyrosine-based activation motif (ITAM; Reth, 1989). The ITAM tyrosines of $\operatorname{Ig} \alpha$ and $\operatorname{Ig} \beta$ control the diverse signaling output of the BCR (Kraus et al., 2004; Gazumyan et al., 2006). Upon phosphorylation, the two tyrosines of the ITAM are bound by the protein tyrosine kinase (PTK) Syk (Grucza et al., 1999). Although Syk controls both proliferation and differentiation of $\mathrm{B}$ and pre-B cells, the Syk substrate SLP-65 (also known as BLNK) mostly promotes differentiation (Herzog et al., 2009). In addition, the BCR provides a survival signal that uses the PI-3 kinase (PI3K) pathway (Srinivasan et al., 2009). Recent findings suggest that Foxo family transcription factors also induce differentiation of preB cells, whereas signals from PI3K negatively control this process by causing the degradation of Foxo proteins (Amin and Schlissel, 2008; Herzog et al., 2008). Interestingly, protein arginine methyltransferase 1 (PRMT1) was found to methylate the Foxo1 protein, thereby inhibiting its phoshorylation and subsequent degradation (Yamagata et al., 2008).

B. Benz and T. Waldmann contributed equally to this paper. T. Waldmann's present address is Faculty of Sciences, University of Konstanz, 78457 Konstanz, Germany.
PRMTs are enzymes that catalyze the transfer of a methyl group from S-adenosyl-methionine to the nitrogen atoms of the arginine guanidinium group (Gary and Clarke, 1998). To date, 12 different PRMTs have been identified (Bedford, 2007; Bedford and Clarke, 2009). Depending on their ability to produce either asymmetric or symmetric dimethylated arginines, they are designated as type I or II enzymes, respectively (Gary and Clarke, 1998). PRMTs not only methylate histones in the nucleus but also substrates in the cytosol, some of which show altered signaling behavior upon methylation (Mowen et al., 2004; Blanchet et al., 2005; Lawson et al., 2007). So far, however, arginine methylation of membrane-bound components has not been described in eukaryotes. We noticed that the Ig $\alpha$ cytoplasmic tail contains a conserved arginine (R198) followed by a glycine (G199), thus resembling the sequence context $(\mathrm{R} G)$ found in PRMT substrate proteins (Najbauer et al., 1993; Blanchet et al., 2006; Bedford, 2007). We show in this paper that R198 of Ig $\alpha$ is constitutively methylated by PRMT1 and that this modification inhibits PI3K signaling while promoting signals leading to $\mathrm{B}$ cell differentiation.

2010 Infantino et al. This article is distributed under the terms of an Attribution-Noncommercial-Share Alike-No Mirror Sites license for the first six months after the publication date (see http://www.rupress.org/terms). After six months it is available under a Creative Commons License (Attribution-Noncommercial-Share Alike 3.0 Unported license, as described at http://creativecommons .org/licenses/by-nc-sa/3.0/). 


\section{RESULTS AND DISCUSSION}

\section{$\lg \alpha$ cytoplasmic tail is methylated by PRMT1}

A comparison of the Ig $\alpha$ tail sequences from several mammals (mouse, human, and bovine) reveals a conserved arginine residue (R198) situated in close proximity to the last ITAM tyrosine (Y193; Fig. 1 A). The emerging role of arginine methylation in lymphocytes prompted us to investigate whether Ig $\alpha$ might be modified by PRMTs.

To test for this, we used a radioactive in vitro methylation assay using the immunopurified, hemagglutinin (HA)-tagged enzymes PRMT1, 3, 5, and 6 with either glutathione S-transferase (GST) or GST-Ig $\alpha$ (mouse cytoplasmic domain) as substrates. After a 1-h reaction, only PRMT1 incorporated methyl groups into proteins of the reaction mix, including a protein of the size of GST-Ig $\alpha$ (Fig. 1 B, top, lane 4, asterisk). Equal loading was confirmed with anti-GST and anti-HA antibodies (Fig. $1 \mathrm{~B}$, middle and bottom), and the activity of all purified PRMT enzymes was verified using histone $\mathrm{H} 2 \mathrm{~A}$ as substrate (Fig. 1 C). This analysis showed that the cytoplasmic tail of $\operatorname{Ig} \alpha$ is a specific substrate of PRMT1 in vitro.

To verify that R198 is the target of PRMT1, we replaced R198 in the tail of Ig $\alpha$ with a lysine (K198). The analysis of GST-Ig $\alpha$ fusion proteins with either WT or K198 mutant tails in the radioactive in vitro methylation assay showed that only the GST-Ig $\alpha$ WT but not the K198 mutant is methylated by PRMT1 (Fig. 1 D, top). Therefore, R198 is the only PRMT1 target site in the Ig $\alpha$ tail sequence.

To test whether Ig $\alpha$ is methylated in B cells, we used ex vivo-cultured pro-B cells derived from the BM of $m b-1^{-/-}$/ B1-8 knockin mice. Because of the deletion of the $m b-1$ gene, these pro-B cells (Ig $\alpha \mathrm{KO}$ ) do not produce Ig $\alpha$ but express the $\mathrm{B} 1-8 \mathrm{H}$ chain from a $\mathrm{V}_{\mathrm{H}} \mathrm{DJ}_{\mathrm{H}}$ knockin allele (Pelanda et al., 2002). Transfection of these pro-B cells with retroviral vectors encoding a $\lambda \mathrm{L}$ chain and a flag-tagged Ig $\alpha$ results in the expression of a BCR that binds to the hapten 4-hydroxy-5iodo-3-nitrophenyl acetyl (NIP; Reth et al., 1978; Meixlsperger et al., 2007).

To monitor arginine methylation of $\operatorname{Ig} \alpha$ more directly, we generated an anti- $\mathrm{R}^{\mathrm{m}}$-Ig $\alpha$ antibody that specifically recognizes the monomethylated form of R198 (Fig. S1). With this antibody, we detected Ig $\alpha$ methylation in Ig $\alpha$ KO B cells stably expressing Ig $\alpha$ WT (Fig. 2 A, top, lane 1). The R198 methylation was prevented by treatment of the B cells with the PRMT1 inhibitor 35605 (Fig. 2 A, top, lane 2; Spannhoff et al., 2007). To obtain more direct evidence that PRMT1 methylates R198 of Ig $\alpha$, we performed a PRMT1 knockdown experiment in HEK293 cells stably expressing a CD8-Ig $\alpha$ chimeric protein. Interestingly, the Ig $\alpha$ cytoplasmic tail is also methylated in these cells, and it is dramatically decreased by small interfering RNA (siRNA) that reduces PRMT1 production (Fig. 2 B).

Using sorted B cell populations from the BM and spleen, we found that PRMT1 is expressed in all B cell subsets examined with the highest amount in pre-B cells (Fig. 2 C, top). The parallel analysis of R198 methylation revealed that $\operatorname{Ig} \alpha$ is methylated in all $\mathrm{BCR}^{+}$cell subsets but not in pre-B

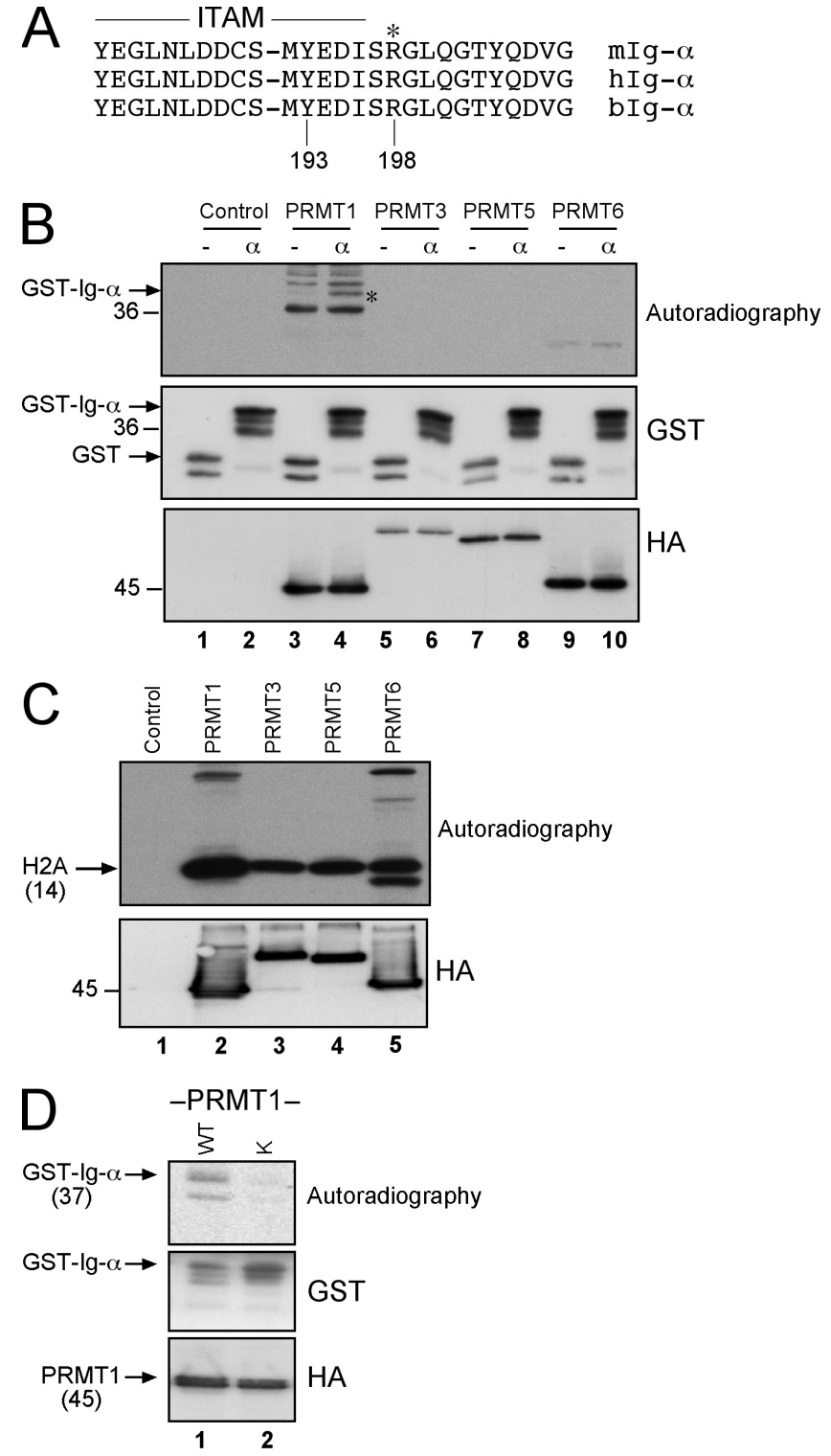

Figure 1. Arginine methylation of the Ig $\alpha$ tail by PRMT1. (A) Sequence alignment of part of the Ig $\alpha$ cytoplasmic tail from mouse $(\mathrm{m})$, human (h), and bovine (b) is depicted. The asterisk shows the position of the conserved arginine. The core region of the ITAM sequence is indicated, as well as the position of tyrosine 193 and arginine 198. (B) In vitro methylation assay of GST and GST-Ig $\alpha$ fusion protein by immunoprecipitated HA-tagged PRMT1, 3, 5, and 6. Arginine methylation of GST-Ig $\alpha(\alpha)$ was detected by autoradiography (lane 4, asterisk). The amount of GST $(-)$ and GST-Ig $\alpha$ proteins was revealed with an anti-GST antibody. An immunoblot with an anti-HA antibody shows the total amount of PRMTs used in the methylation reaction. (C) In vitro methylation assay of histone $\mathrm{H} 2 \mathrm{~A}$ by immunoprecipitated HA-tagged PRMTs. Methyl- ${ }^{3} \mathrm{H}$ incorporation was detected by autoradiography. The total amount of PRMTs was determined with an anti-HA antibody. (D) GST-Ig $\alpha$ (WT) and GST-Ig $\alpha$ K198 mutant $(K)$ were used in the in vitro methylation assay by immunoprecipitated HA-tagged PRMT1. Autoradiography shows methylated Ig $\alpha$ (lane 1). Equal loading was determined by immunoblotting with anti-GST and anti-HA antibodies. Apparent molecular weights are indicated. One representative experiment out of three is shown. 
cells (Fig. 2 D, top). It has been reported that the pre-BCR constitutively produces an autonomous ligand-independent signal (Meixlsperger et al., 2007). The finding that Ig $\alpha$ methylation was detected in B cells but not in pre-B cells suggests that the R198 methylation has an inhibitory role.

\section{BCR activation alters $\lg \alpha$ arginine methylation}

To investigate whether the tail of $\operatorname{Ig} \alpha$ is also methylated upon $\mathrm{B}$ cell activation, we reconstituted Ig $\alpha \mathrm{KO}$ cells with both Ig $\alpha$ WT or Ig $\alpha$ K198 mutant and L chain. A FACS analysis verified that transfectants producing either Ig $\alpha$ WT or the Ig $\alpha$ K198 mutant express similar amounts of BCR on the surface (Fig. S2, top).

The transfected B cells were cultured for $3 \mathrm{~h}$ with L[methyl- ${ }^{3} \mathrm{H}$ ]methionine in the presence of protein synthesis inhibitors. The cells were then either left unstimulated or stimulated for different times $(2.5-30 \mathrm{~min})$ with NIP-BSA (Fig. 2 E). The Ig $\alpha$ proteins in the total cellular lysate of these cells were immunopurified with anti-flag antibodies and analyzed for methyl- ${ }^{3} \mathrm{H}$ incorporation by autoradiography (Fig. 2 E, top). Radioactive methyl groups were predominantly incorporated into Ig $\alpha$ WT and to a much lesser extent into the Ig $\alpha$ K198 mutant. The weak radioactivity of the Ig $\alpha$ K198 mutant may be caused by an incomplete inhibition of protein synthesis that resulted in some incorporation of $\left[{ }^{3} \mathrm{H}\right]$ methionine in newly synthesized Ig $\alpha$. Interestingly, the methylation of $\operatorname{Ig} \alpha \mathrm{WT}$ was strongest in nonstimulated B cells, declined 2.5 and 5 min after BCR ligation, and reappeared after $30 \mathrm{~min}$ of exposure of B cells to NIP-BSA (Fig. 2 E, top). We controlled for similar amounts of precipitated Ig $\alpha$ protein with an antiflag antibody (Fig. 2 E, middle). Furthermore, similar amounts of methylated proteins in the total cellular lysates of the cells demonstrated equal ${ }^{3} \mathrm{H}$ incorporation (Fig. $2 \mathrm{E}$, bottom). This is in line with equal expression of PRMT1 in untransfected as well as in Ig $\alpha$-expressing B cells (Fig. S3, top). The timecourse experiment was then repeated using the specific anti$\mathrm{R}^{\mathrm{m}}$-Ig $\alpha$ antibody to detect R198 methylation in the total cellular lysate of WT or mutant Ig $\alpha$-producing B cells. Again,
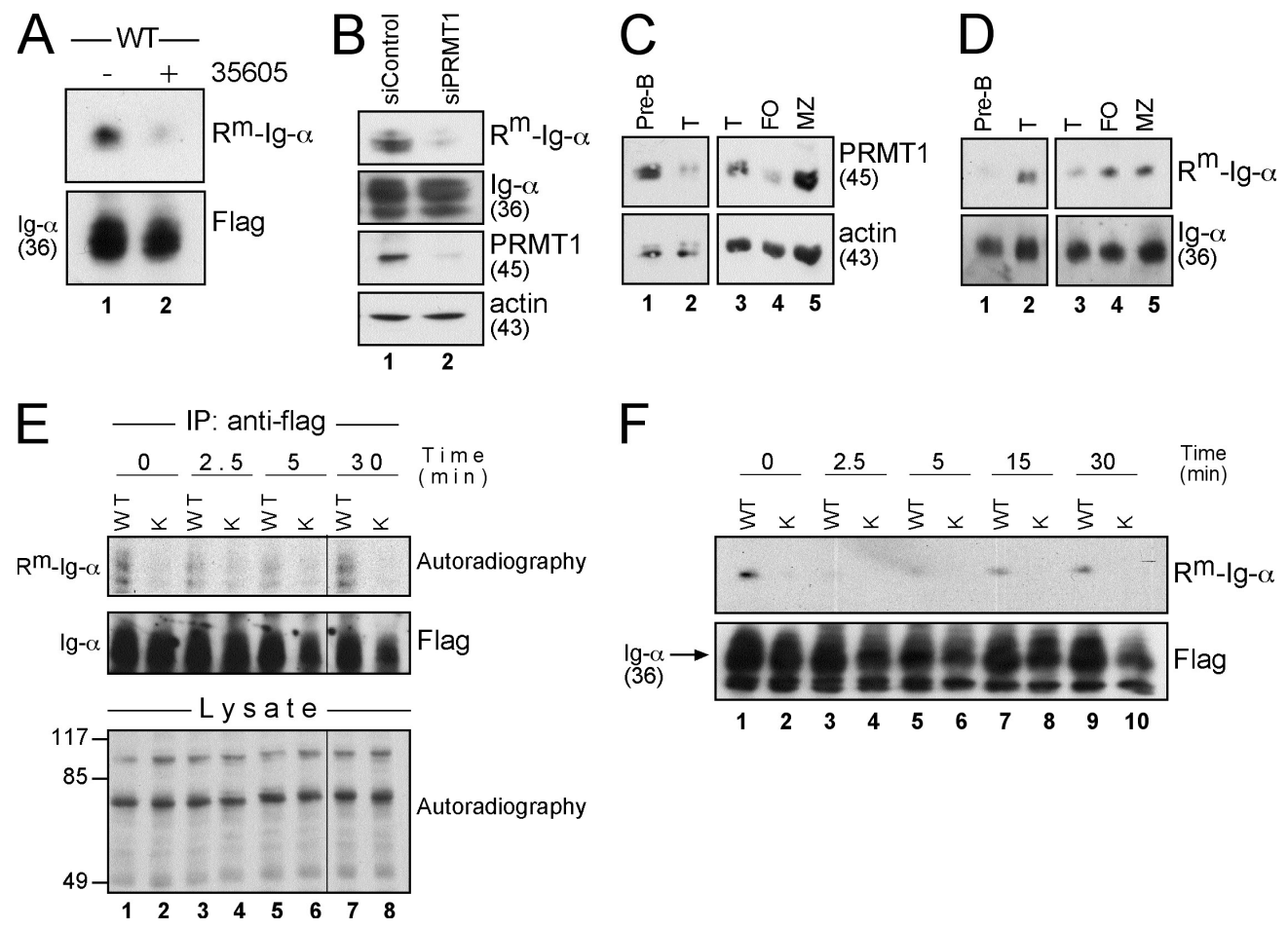

Figure 2. Alteration of Ig $\alpha$ arginine methylation upon BCR activation. (A) B cells containing WT Ig $\alpha$ were treated without $(-)$ or with $(+) 50 \mu M$ PRMT1 inhibitor (35605). Ig $\alpha$ arginine methylation was detected with an anti- $\mathrm{R}^{\mathrm{m}}-\lg \alpha$ antibody (top). An equal amount of Ig $\alpha$ is shown by immunoblotting with an anti-flag antibody. (B) HEK293 cells stably expressing CD8-Ig $\alpha$ were transfected with nontargeting siRNA (lane 1) or siPRMT1 (lane2). The whole-cell lysates were analyzed by immunoblotting with anti-R ${ }^{m}-\lg \alpha$, anti-lg $\alpha$, anti-PRMT1, and antiactin. (C and D) Western blot analysis of primary cells sorted from BM (pre-B cells) and spleen (transitional [T], follicular [FO], and marginal zone [MZ]). PRMT1 protein levels and Ig $\alpha$ arginine methylation are indicated with an anti-PRMT1 antibody (C) and with an anti- $R^{m}-\lg \alpha$ antibody (D), respectively. A loading control is shown with antiactin (C) and with anti-Ig $\alpha$ (D) antibodies. (E) In vivo methylation assay. B cells carrying WT or mutant Ig $\alpha$ were labeled with L-[methyl- $\left.{ }^{3} \mathrm{H}\right]$ methionine in the presence of protein synthesis inhibitors. Cells were stimulated as indicated with NIP-BSA for different time points. Ig $\alpha$ was further immunoprecipitated (IP) with an anti-flag antibody, and arginine methylation $\left(\mathrm{R}^{\mathrm{m}}-\lg \alpha\right)$ was revealed by autoradiography (top). The total amount of immunoprecipitated Ig $\alpha$ was detected with an anti-flag antibody (middle). Equal methylation was determined by measuring ${ }^{3} \mathrm{H}$ incorporation in the total lysate (bottom). In each panel, the vertical black lines separate two different parts of the same membrane. (F) B cells expressing WT or mutant lg $\alpha$ were activated with antigen for the indicated times. Ig $\alpha$ arginine methylation was detected by immunoblotting with an anti- $\mathrm{R}^{\mathrm{m}}-\lg \alpha$ antibody. The total amount of $\lg \alpha$ was measured with an anti-flag antibody. Apparent molecular weights are indicated. Data are representative of three independent experiments. 
Ig $\alpha$ R198 methylation was high in resting B cells, reduced 2.5 and $5 \mathrm{~min}$ after BCR activation, and reappeared at later time points (Fig. 2 F, top). These data, together with the absence of Ig $\alpha$ methylation in pre-B cells, suggest that R198 methylation is a feature of the BCR on resting $\mathrm{B}$ cells.

To directly test for Ig $\alpha-P R M T 1$ interaction, we used the Duolink in situ proximity ligation assay (PLA). Intriguingly, PRMT1 appeared to be most strongly associated with Ig $\alpha$ before activation (Fig. $3 \mathrm{~A}$, top right). At $2.5 \mathrm{~min}$ after BCR activation, PRMT1 partially dissociated from Ig $\alpha$ to further reassociate with it at later time point (Fig. $3 \mathrm{~A}$, bottom). The time course of Ig $\alpha-P R M T 1$ interaction correlates well with the kinetics of Ig $\alpha$ methylation. No signal was visible in the Ig $\alpha \mathrm{KO}$ control cells (Fig. 3 A, top left). The number of dots per cell were counted and plotted with the relative statistical calculations (Fig. 3 B). The specificity of this assay was also confirmed by analyzing the Ig $\alpha$-Syk interaction, which occurred only after BCR activation (Fig. 3, C and D).

\section{Arginine methylation of $\lg \alpha$ modulates BCR activation}

To examine the role of $\operatorname{Ig} \alpha$ arginine methylation in BCR signaling, we compared the behavior of B cells expressing either Ig $\alpha$ WT or the Ig $\alpha$ K198 mutant. One of the earliest events after the engagement of the BCR is the activation of the PTKs Syk and Lyn and the phosphorylation of several PTK substrates, including Ig $\alpha$. A time-course experiment monitoring tyrosine phosphorylation after stimulation of the BCR with NIP-BSA did not show any major difference between B cells expressing WT or mutated Ig $\alpha$ (Fig. 4 A, top). However, by using site-specific antibodies detecting either the phosphorylation of Y630 in the C-terminal tail of Syk (Kulathu et al., 2008) or phosphorylation of S473 in the serine/threonine kinase $\mathrm{AKT} / \mathrm{PKB}$, we found that both proteins are more strongly phosphorylated in B cells expressing a BCR with the Ig $\alpha$ K198 mutant than in those carrying Ig $\alpha$ WT (Fig. 4 B, top and middle). Interestingly, after 30 min of BCR stimulation, the phosphorylation of these proteins declines, whereas arginine methylation of Ig $\alpha$ reappears (compare Fig. 2 E, Fig. 2 F, and Fig. 4 B). This inverse correlation between phosphorylation and arginine methylation suggests that the methylation of R198 of Ig $\alpha$ plays a negative regulatory role for $\mathrm{B}$ cell activation.

The R198 is situated only 5 aa after the last ITAM tyrosine (Y193), and it is feasible that methylation of R198 may interfere with the binding of Syk to the BCR. To examine this, we purified the BCR containing either Ig $\alpha$ WT or Ig $\alpha$ K198 mutant at different time points after B cell activation and found that $2-5 \mathrm{~min}$ after stimulation, Syk is more
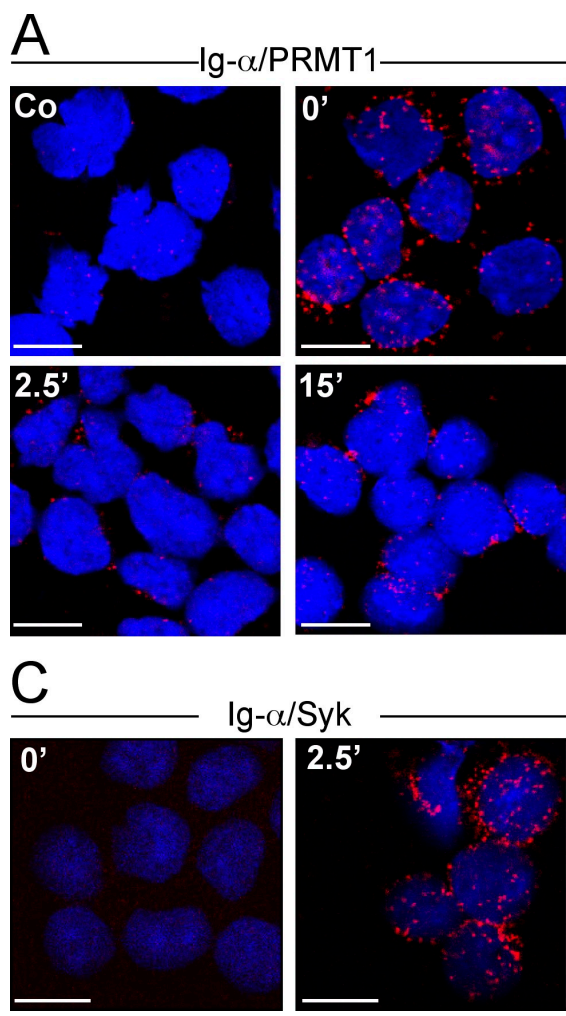
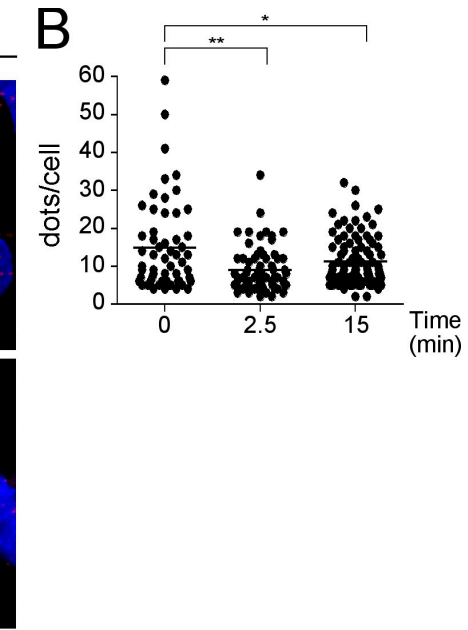

Figure 3. PRMT1-Ig $\alpha$ interaction is regulated upon BCR activation. (A) Ig $\alpha$-PRMT1 binding was visualized in B cells using in situ PLA. Ig $\alpha$ KO cells (Co) reconstituted with Ig $\alpha$ WT were stimulated with NIP-BSA for the indicated time points. Bars, $10 \mu \mathrm{m}$. (B) The dots per cell were counted and plotted. Each data point represents a single cell and the bars indicate mean values $\left({ }^{*}, \mathrm{P}<0.04\right.$; and ${ }^{* *}, \mathrm{P}<0.01$ vs. nonactivated $\mathrm{B}$ cells). (C) Ig $\alpha$ WT-expressing $\mathrm{B}$ cells were activated with antigen and Ig $\alpha$-Syk interaction was detected using in situ PLA. Bars, $10 \mu \mathrm{m}$. (D) Each point is a single cell. Bars indicate mean values ${ }^{* *}, P<0.01$ vs. nonactivated B cells). Nuclei were stained with Hoechst. The experiment was repeated two times with similar results. 
strongly associated with the mutant than with the WT BCR (Fig. 4 C, top). These data support the notion that the methylation of R198 can modulate BCR signaling.

\section{$\lg \alpha$ arginine methylation negatively controls $\mathrm{Ca}^{2+}$ signaling}

$\mathrm{B}$ cells respond to $\mathrm{BCR}$ ligation with a rapid increase in the intracellular $\mathrm{Ca}^{2+}$ concentration (Scharenberg et al., 2007). We compared the $\mathrm{Ca}^{2+}$ response of NIP-BSA-stimulated B cells expressing either WT or an Ig $\alpha$ K198 mutant BCR. The $\mathrm{Ca}^{2+}$ influx was clearly higher in the $\mathrm{B}$ cells with the mutant BCR (Fig. 5 A). The same was true for cells expressing a mutant pre-BCR and stimulated with anti-IgM antibodies (Fig. 5 D). The mutant pre-BCR was expressed at similar levels to the WT pre-BCR (Fig. S2, bottom). The increased $\mathrm{B}$ cell response could be caused by the $\mathrm{R} \rightarrow \mathrm{K} 198$ mutation itself or by the absence of Ig $\alpha$ methylation. To test this, we stimulated cells expressing the WT BCR with NIP-BSA in the absence or presence of the PRMT1 inhibitor 35605 and monitored the calcium flux of the stimulated B cells. Interestingly, the inhibition of PRMT1 in stimulated cells expressing Ig $\alpha \mathrm{WT}$ resulted in a calcium response increased to a level similar to that seen in Ig $\alpha$ K198 mutant B cells (Fig. 5 B). This finding indicates that it is the absence of methylation and not the mutation of $\operatorname{Ig} \alpha$ per se that increases the $\mathrm{Ca}^{2+}$ response in mutant B cells. Furthermore, when the PRMT1 inhibitor was applied to B cells expressing the Ig $\alpha$ K198 mutant, no further augmentation in calcium flux was observed upon stimulation (Fig. 5 C), suggesting that the methylation of Ig $\alpha$ rather than that of other PRMT1 substrates controls the intensity of the $\mathrm{Ca}^{2+}$ signal.

\section{$\lg \alpha$ arginine methylation supports $B$ cell differentiation}

The cytokine IL-7 is an important growth factor for mouse pre-B cells. The removal of IL-7 from pre-B cell cultures results in growth arrest of the pre-B cells and their increased differentiation, as indicated by L chain gene ( $\kappa$ followed by $\lambda$ ) assembly and the expression of a BCR on the surface of these cells (Rolink et al., 1991; Flemming et al., 2003). By comparing the amount of $\mathrm{BCR}^{+}$cells generated after withdrawal of IL-7 from pre-B cell cultures expressing either Ig $\alpha$ WT or the Ig $\alpha \mathrm{K} 198$ mutant, we noticed that the latter cells display a partial block in B cell differentiation (Fig. 5 E). $5 \mathrm{~d}$ after IL-7 removal, the Ig $\alpha \mathrm{WT}$ cultures contained $14 \%$ of $\mathrm{BCR}^{+}$cells, whereas parallel cultures of mutant pre-B cells contained only $5.5 \% \mathrm{BCR}^{+}$cells (Fig. 5 E, bottom; and Fig. S4 A). To test whether arginine methylation mediated by PRMT1 was

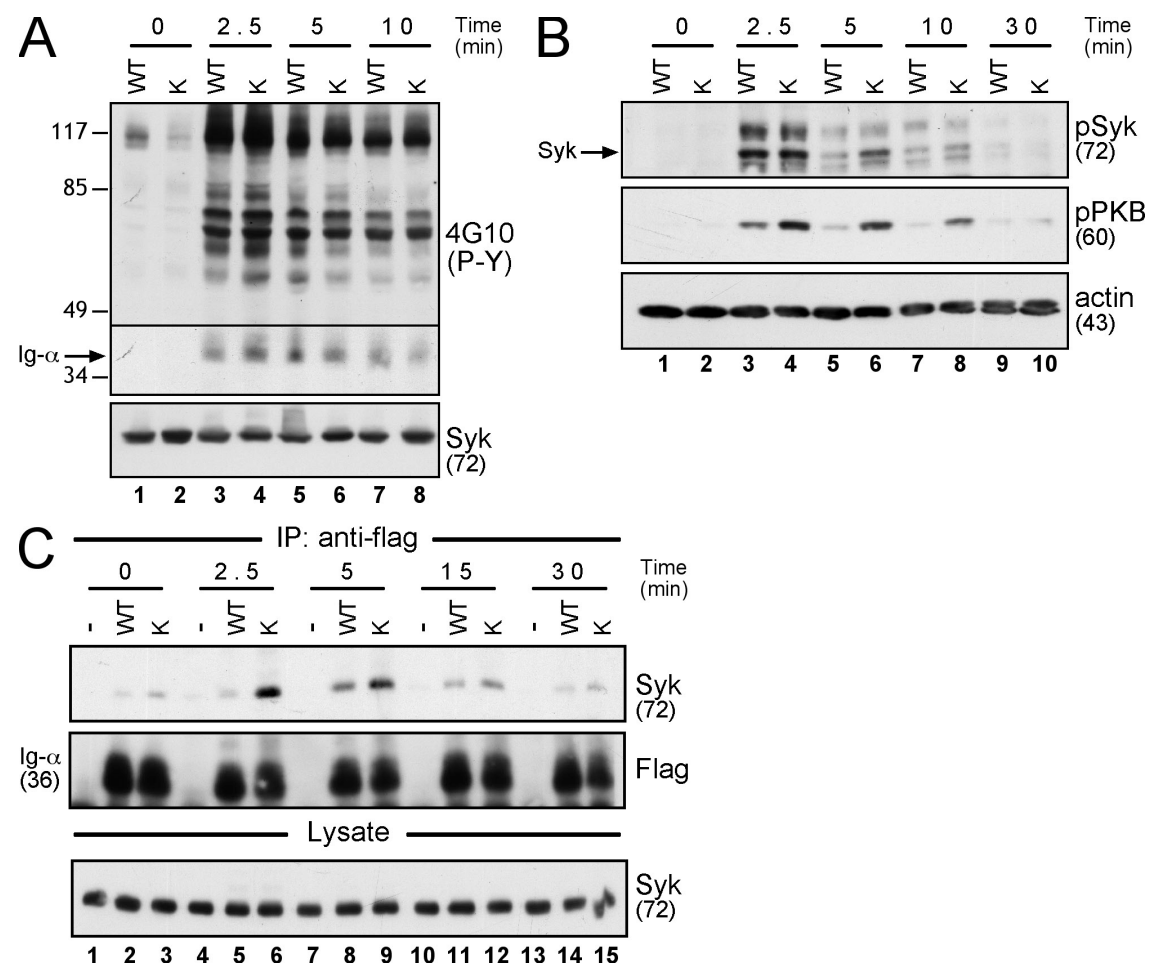

Figure 4. $\lg \alpha$ methylation plays a negative role in BCR signaling. $\lg \alpha$ KO cells reconstituted with WT or mutant $\lg \alpha(K)$ and $\lambda 1$ were stimulated with NIP-BSA for the indicated time points. (A) Western blot analysis of whole-cell lysates is shown with antiphosphotyrosine (4G10) and anti-Syk antibodies. (top) A horizontal black line separates the short exposure (top part) from the long exposure (bottom part). (B) B cells expressing WT or mutant lg $\alpha$ were activated with antigen for the indicated times. Whole-cell lysates were immunoblotted with anti-pSyk (Y630) and anti-pPKB (S473) antibodies. Equal loading is shown with the antiactin antibody. (C) $\lg \alpha \mathrm{KO}(-)$ cells carrying WT or mutant $\lg \alpha(\mathrm{K})$ and $\lambda 1$ were stimulated with NIP-BSA for the indicated times. Ig $\alpha$ was further immunoprecipitated (IP) with an anti-flag antibody and Syk was detected with an anti-Syk antibody (top). The total amount of immunoprecipitated Ig $\alpha$ was revealed with an anti-flag antibody. The amount of Syk in the whole-cell lysate is shown by immunoblotting with an anti-Syk antibody (bottom). Apparent molecular weights are indicated. Similar results were obtained in three independent experiments. 


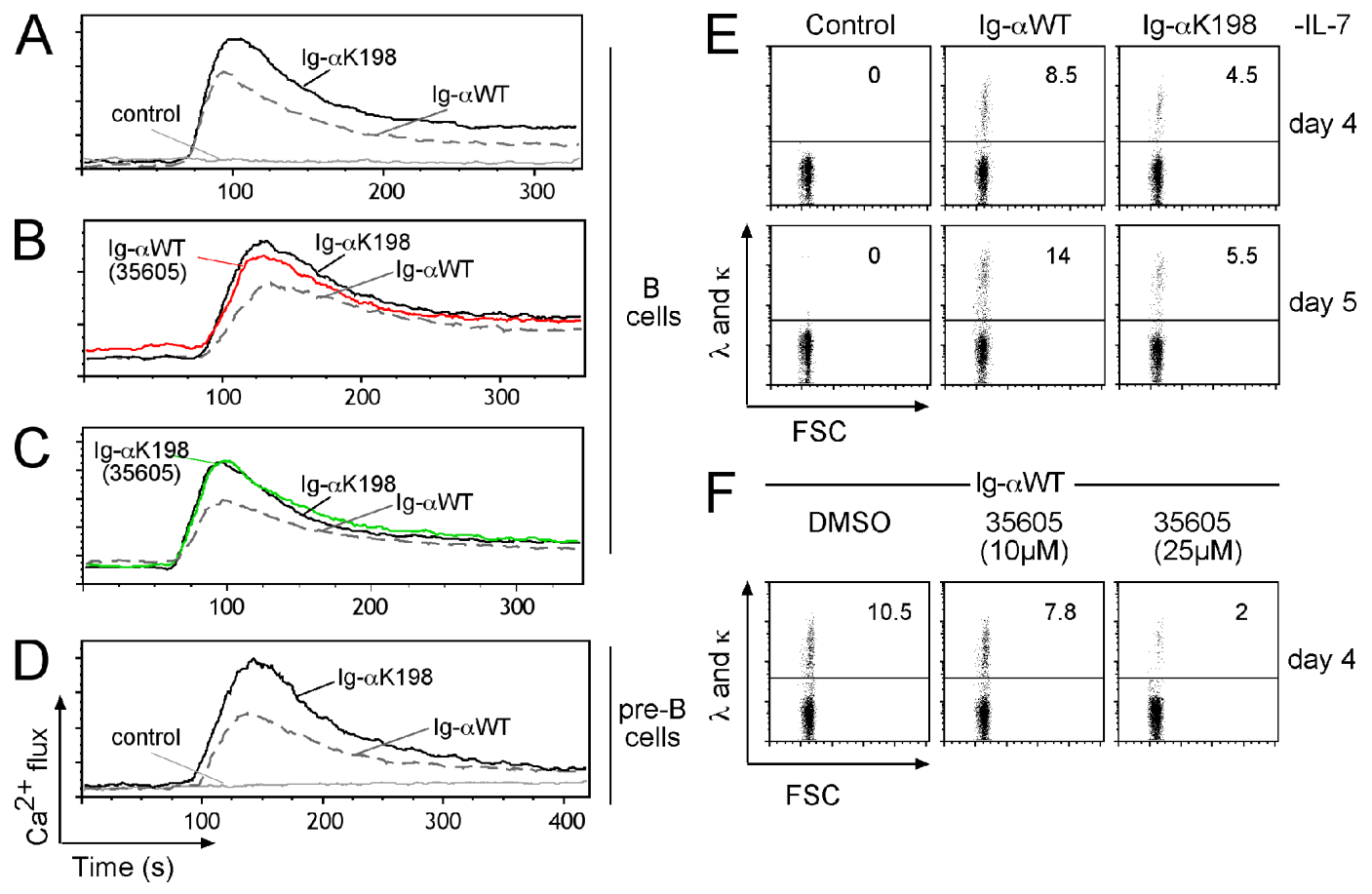

Figure 5. Increased intracellular $\mathrm{Ca}^{2+}$ release and partial differentiation impairment in cells expressing mutant Ig $\alpha$. (A) Intracellular $\mathrm{Ca}^{2+}$ was measured in B cells expressing WT or mutant Ig $\alpha$ (Ig $\alpha$ K198) after BCR antigen stimulation. The control represents the Ig $\alpha$ KO cells. (B and C) Before BCR activation, B cells were treated with $50 \mu \mathrm{M}$ PRMT1 inhibitor (35605). (D) Pre-B cells carrying WT or mutant Ig $\alpha$ (Ig $\alpha$ K198) were stimulated with anti-lgM and $\mathrm{Ca}^{2+}$ flux was measured. The control corresponds to Ig $\alpha \mathrm{KO}$ cells. (E) Differentiation of pre-B cells was induced by withdrawal of IL-7. 4 and $5 \mathrm{~d}$ after IL-7 withdrawal, $\kappa$ and $\lambda$ expression was measured by FACS analysis. (F) Pre-B cells expressing WT Ig $\alpha$ were cultured without IL-7 in the absence or presence of PRMT1 inhibitor (35605). On day 4, the amount of $\kappa$ and $\lambda$ positive cells was detected by FACS analysis. The numbers represent the percentage of cells expressing $L$ chain. Dead cells stained with propidium iodide were excluded from the analysis. Comparable data were obtained in three independent experiments. FSC, forward scatter.

required for efficient $\mathrm{B}$ cell differentiation, we cultured Ig $\alpha \mathrm{WT}$ pre-B cells for $4 \mathrm{~d}$ without IL-7 in the presence of different doses of the PRMT1 inhibitor 35605 (Fig. 5 F; and Fig. S4 B). At the highest dose of inhibitor, the number of $\mathrm{BCR}^{+}$cells was reduced fivefold.

To test the in vivo function of R198 in B cell development, we reconstituted pro- $\mathrm{B}$ cells derived from Ig $\alpha \mathrm{KO}$ mice with an expression vector either for GFP, Ig $\alpha$ WT-IRES-GFP, or Ig $\alpha$ K198-IRES-GFP. The cells were sorted for GFP expression and transferred into RAG-2 $2^{-/-} / \gamma^{-/-}$mice. The developing $\mathrm{B}$ cells were analyzed $10 \mathrm{~d}$ later. In the spleen, most of the pre-B cells expressing WT Ig $\alpha$ developed into $\mathrm{BCR}^{+}$cells $\left(67.7 \% \mathrm{IgM}^{+}\right)$, whereas pre-B cells carrying Ig $\alpha \mathrm{K} 198$ produced fewer IgM ${ }^{+}$cells (19.7\%; Fig. 6 A). Similarly, in the BM, although the majority of WT Ig $\alpha$-expressing pre-B cells became $\operatorname{IgM}^{+}$, only a few of the mutant cells developed into $\operatorname{IgM}^{+}$ cells (Fig. 6 B). In comparison to WT cells, a higher amount of Ig $\alpha$ K198-expressing cells was found in the BM (Fig. 6 C, right). In the spleen, this situation was reversed (Fig. $6 \mathrm{C}$, left). This finding shows that Ig $\alpha$ methylation is important for regulating $\mathrm{B}$ cell differentiation.

The expression of a functional pre-BCR is required for both the proliferation and the differentiation of pre- $\mathrm{B}$ cells. Thus, signals from the same receptor result in opposing cellular programs. Recently, it was discovered that Foxo family transcription factors promote the differentiation of pre-B cells and that signals processed through Syk and the PI3K pathways counteract this activity by degrading Foxo proteins (Amin and Schlissel, 2008; Herzog et al., 2008). Our finding that the $\mathrm{R} \rightarrow \mathrm{K} 198$ mutation increases Syk and PI3K signaling of the $\mathrm{BCR}$ and reduces the differentiation signal of the pre-BCR is in line with these data.

Ig $\alpha$ arginine methylation appears to damper signals from the BCR. In pre-B cells, Ig $\alpha$ methylation is absent and the pre-BCR signals constitutively. Because receptor editing or B cell deletion removes cells with autoreactive BCR (Nemazee and Bürki, 1989; Gay et al., 1993), it is feasible that stronger signaling from BCR lacking Ig $\alpha$ arginine methylation could also lead to negative selection. This may explain why the development of pre-B cells carrying IgaK198 mutant is impaired.

Finally, the dynamic changes in arginine methylation of Ig $\alpha$ we detected after BCR stimulation suggest that not only arginine methylases but also arginine demethylases might modulate B cell activation. It is feasible that upon activation, the dissociation of PRMT1 from the BCR allows the recruitment of an arginine demethylase. Indeed, the Ig $\alpha-P R M T 1$ interaction corresponds to the kinetics of 


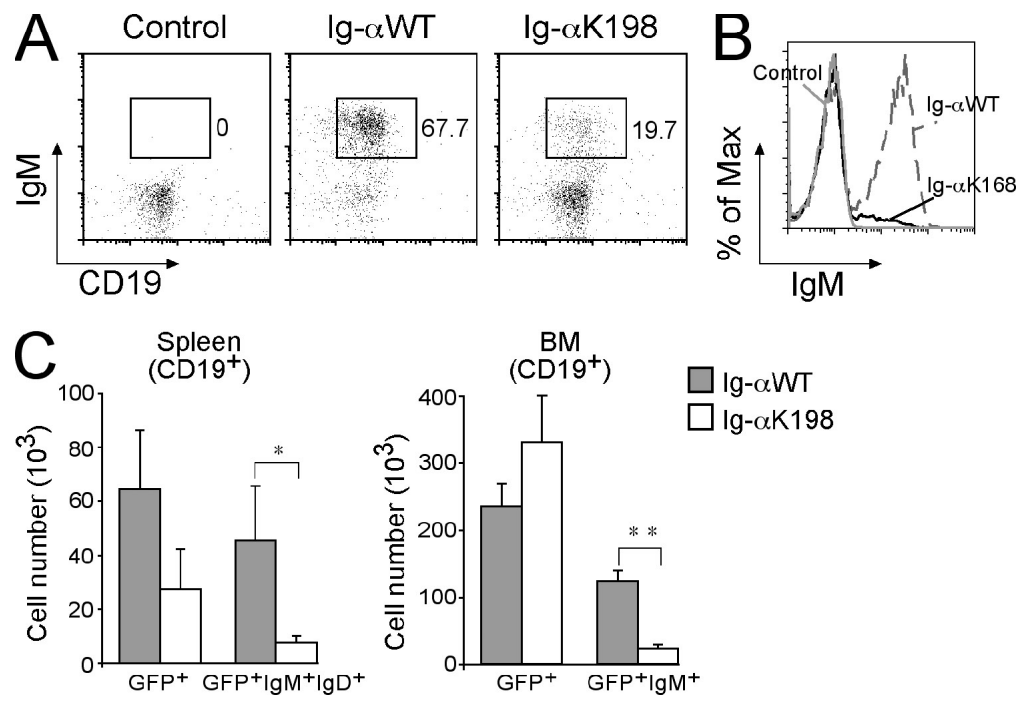

Figure 6. Ig $\alpha$ methylation supports B cell development. Ig $\alpha$ KO cells (control) infected with Ig $\alpha$ WT or lg $\alpha$ K 198 were intravenously transferred into RAG-2-I- $/ \gamma c^{-1-}$ mice. $10 \mathrm{~d}$ after injection, the resulting B cells were analyzed by flow cytometry. (A) Splenic B cells are shown in the dot plot with antiCD19 and anti-IgM antibodies. The cells were gated on the GFP+ population. The percentages of $\mathrm{CD}^{+} \mathrm{g}^{+} \mathrm{IgM}^{+}$cells are indicated. (B) The histogram shows the amount of BCR (IgM) on the surface of GFP+CD19+ BM cells. (C) The numbers of transferred cells found in the spleen (left) and in the BM (right) are plotted. Error bars indicate means + SEM (each with three mice; ${ }^{*}, P<0.04 ;{ }^{* *}, P<0.01$ ). The cell transfer was performed two times using three mice per group per experiment. In these two sets of experiments, the cells were separately infected and sorted based on GFP expression.

Ig $\alpha$ methylation. The identification and characterization of an arginine demethylase is likely to shed light on the regulation of BCR signaling.

\section{MATERIALS AND METHODS}

Mice. RAG-2 ${ }^{-/-} / \gamma^{-/-}$mice (Colucci et al., 1999) were used for the in vivo differentiation assay. Pro-B cells derived from the $m b-1^{-1-} / \mathrm{B} 1-8$ knockin mice were retrovirally transduced with the respective vectors and injected intravenously into RAG-2 $2^{-/-} / \mathrm{yc}^{-/-}$mice. $10 \mathrm{~d}$ later, mice were sacrificed to analyze spleen and BM. BALB/c (WT), $m b-1^{-/} / \mathrm{B} 1-8$ knockin, and RAG-2 $2^{-1-} / \gamma^{-1-}$ mice were bred at the animal facility of the Max-PlanckInstitute for Immunobiology. Animal experiments were performed as approved by the Regieriungspräsidium Freiburg.

Cell culture, transfection, and retroviral transduction. The pro-B cell line was established from $m b-1^{-/-} / \mathrm{B} 1-8$ knockin BM cells cultured in Iscove's medium containing 10\% heat-inactivated FCS (PAN Biotech $\mathrm{GmbH}$ ), $100 \mathrm{U} / \mathrm{ml}$ penicillin/streptomycin (Invitrogen), $50 \mu \mathrm{M}$ 2-mercaptoethanol (EMD), and $0.5 \mathrm{ng} / \mathrm{ml} \mathrm{IL-7} \mathrm{derived} \mathrm{from} \mathrm{the} \mathrm{supernatant} \mathrm{of} \mathrm{a} \mathrm{J558L} \mathrm{cell} \mathrm{line}$ transfected with a vector encoding mouse IL-7. Retroviruses (pMOWSIRES-CD8 [Herzog and Jumaa, 2007] and pMOWS-flag-Ig $\alpha$-IRES-CD8, pMOWS-flag-Ig $\alpha-K-I R E S-C D 8$, pMIG-IRES-GFP, pMIG-flag-Ig $\alpha$ IRES-GFP, pMIG-flag-Ig $\alpha-K-I R E S-G F P$, and pMOWS- $\lambda 1$ [Meixlsperger et al., 2007]) were generated by calcium chloride transfection of the Phoenix retroviral producer cell line. Supernatants containing retroviruses were harvested after $2 \mathrm{~d}$. Viral infection of $2 \times 10^{5}$ pro-B cells was performed by the addition of $500 \mu \mathrm{l}$ of supernatant followed by centrifugation at $1,800 \mathrm{rpm}$ for $3 \mathrm{~h}$ at $37^{\circ} \mathrm{C}$. Cells were expanded and sorted based on CD8 or GFP expression. $\lambda 1$ expression was selected with $1 \mu \mathrm{g} / \mathrm{ml}$ puromycin. The pcDNA3.1PRMT plasmids were used to transfect HEK293 cells with the calcium phosphate method and were further selected in medium-containing G418.

Plasmids. A cDNA encoding an N-terminal flag-tag mouse Ig $\alpha$ (Rolli et al., 2002) was inserted into pMOWS-IRES-CD8. The R198K mutation was introduced by sequential PCR steps. PCR fragments were cloned into a DraIII/XhoI-digested $\mathrm{pD}$-flag-Ig $\alpha$ plasmid and subsequently transferred into
pMOWS-IRES-CD8 or pMIG-IRES-GFP. The plasmids pRp261 and pRp261-Ig $\alpha$ were used to generate the recombinant proteins GST and GST-Ig $\alpha$, respectively. The $\mathrm{pR}$ 261-Ig $\alpha \mathrm{K} 198$ mutant vector was generated by PCR. PRMT1, 3, 5, and 6 were cloned in the pcDNA3.1 vector containing one flag- and two tandem HA-tags at the $\mathrm{N}$ terminus.

siRNA. HEK293 cells were stably transfected with CD8-Ig $\alpha$ (this chimeric protein contains the extracellular and the transmembrane region of the mouse CD8 $\alpha$ fused with the cytoplasmic tail of mouse Ig $\alpha$ ). The siRNAs ( $80 \mathrm{nM}$ final concentration) were complexed with Cellfectin (Invitrogen) in Optimem medium for $25 \mathrm{~min}$ and were applied to the cells. After 4 h, DMEM (supplied with 10\% FCS without phenol red and antibiotics) was added and incubated for $72 \mathrm{~h}$. To target human PRMT1, the siRNA oligonucleotide was purchased from Santa Cruz Biotechnology, Inc. The nontargeting control siRNA was from Thermo Fisher Scientific.

Antibodies and reagents. The following antibodies were used for Western blotting: antiphosphotyrosine (4G10), anti-GST (Bethyl Laboratories, Inc.), anti-HA (Roche), anti-flag (Sigma-Aldrich), antiactin, and anti-Syk (N19; Santa Cruz Biotechnology, Inc.), anti-pY630-Syk (Kulathu et al., 2008), anti-PRMT1, and anti-pS473-PKB (Cell Signaling Technology). For immunoprecipitation, the following antibodies were used: anti-flag (SigmaAldrich) and anti-HA (12CA5). The anti-methyl-Ig $\alpha$ (anti- $\left.\mathrm{R}^{\mathrm{m}}-\operatorname{Ig} \alpha\right)$ antibody was generated by immunizing rabbit with mono-methyl-Ig $\alpha$ peptide (190-202 aa). Affinity purification of the antiserum was performed to produce a methyl-specific antibody (Eurogentec).

W. Sippl (Martin-Luther-Universität Halle-Wittenberg, Halle, Germany) provided the PRMT1 inhibitor (NSC 35605). The inhibitor was obtained from the National Cancer Institute/Developmental Therapeutics Program Open Chemical Repository (available at http://dtp.cancer.gov).

In vitro methylation assays. $2 \mu \mathrm{g}$ GST and GST Ig $\alpha$ recombinant proteins were incubated with $10 \mu \mathrm{l}$ of immunoprecipitated PRMT enzymes. $0.5 \mu \mathrm{Ci} \mathrm{S}$-adenosyl-L-[methyl- $\left.{ }^{3} \mathrm{H}\right]$ methionine (the methyl group donor) was added and the reaction was incubated for $1 \mathrm{~h}$ at $30^{\circ} \mathrm{C}$. The samples were loaded on a gel and blotted onto a nitrocellulose membrane. 
In vivo methylation assay. $2 \times 10^{6} \mathrm{~B}$ cells $/ \mathrm{ml}$ were incubated in RPMI 1640 complete medium (10\% heat-inactivated FCS, $100 \mathrm{U} / \mathrm{ml}$ penicillin/ streptomycin [Invitrogen], $50 \mu \mathrm{M}$ 2-mercaptoethanol, and IL-7, without methionine) containing $20 \mu \mathrm{g} / \mathrm{ml}$ chloramphenicol and $100 \mu \mathrm{g} / \mathrm{ml}$ cycloheximide (Sigma-Aldrich) for $30 \mathrm{~min}$. The medium was replaced with RPMI 1640 complete medium containing $10 \mu \mathrm{Ci} / \mathrm{ml}$ of L-[methyl- $\left.{ }^{3} \mathrm{H}\right]$ methionine (GE Healthcare). The cells were incubated for an additional $3 \mathrm{~h}$ in the presence of the methyl group donor and the same protein synthesis inhibitors.

Isolation of primary B cells. B cells were isolated from the spleen of WT $\mathrm{BALB} / \mathrm{c}$ mice by depletion with an anti-CD43 antibody coupled to magnetic microbeads using an automated magnetic cell sorter (autoMACS; Miltenyi Biotec). The negative fraction obtained by magnetic sorting was stained with CD19-FITC, CD93-allophycocyanin (APC), CD21-PE, and CD23-PE-Cy7 (eBioscience). Transitional $\left(\mathrm{CD} 19^{+} \mathrm{CD} 93^{+}\right)$, follicular $\left(\mathrm{CD} 19^{+}\right.$ $\left.\mathrm{CD} 93^{-} \mathrm{CD} 23^{+} \mathrm{CD} 21^{\text {low }}\right)$, and marginal zone $\left(\mathrm{CD} 19^{+} \mathrm{CD} 93^{-} \mathrm{CD} 23^{-} \mathrm{CD} 21^{+}\right)$ B cells were sorted by a FACSAria (BD; Matthias and Rolink, 2005). All B cell populations were $99 \%$ pure. Pre-B cells $\left(\mathrm{CD} 19^{+} \mathrm{CD} 25^{+}\right)$, derived from $\mathrm{BM}$ of $\mathrm{WT} \mathrm{BALB} / \mathrm{c}$ mice, were sorted using the following antibodies: CD19-FITC and CD25-PE (eBioscience).

In situ PLA. Unstimulated or stimulated B cells were centrifuged onto microscope slides fixed with $2 \%$ PFA for $30 \mathrm{~min}$ on ice, permeabilized with $0.5 \%$ saponin for $3 \mathrm{~min}$, and stained according to the manufacturer's instructions with the Duolink kit (Olink Bioscience). The antibody combinations used were rabbit-anti-Ig $/$ mouse-anti-PRMT1 (Eurogentec and Abcam, respectively) or rabbit-anti-Ig $\alpha /$ mouse-anti-Syk (4D10; Eurogentec and Santa Cruz Biotechnology, Inc., respectively). The nuclei were stained with Hoechst. The images of the cells were taken with a confocal microscope (SP2; Leica), and they have been acquired with one z-plane. The image analysis was performed with the freeware BlobFinder (available at http://www.cb.uu.se/ amin/BlobFinder/).

Measurement of $\mathrm{Ca}^{2+}$ release. $3 \times 10^{6}$ cells were incubated with $5 \mu \mathrm{g} / \mathrm{ml}$ Indo- 1 and $0.5 \mu \mathrm{g} / \mathrm{ml}$ pluronic acid (Invitrogen) in Iscove's medium supplemented with $1 \% \mathrm{FCS}$ at $37^{\circ} \mathrm{C}$ for $45 \mathrm{~min}$. The loaded cells were resuspended in Iscove's medium containing $1 \% \mathrm{FCS}$, and $\mathrm{Ca}^{2+}$ release was induced by the addition of $1 \mathrm{ng} / \mathrm{ml}$ NIP-conjugated BSA (15 haptens per BSA molecule; Biosearch Technologies) or $10 \mu \mathrm{g} / \mathrm{ml}$ of goat anti-mouse IgM (SouthernBiotech). $\mathrm{Ca}^{2+}$ flux was measured with an LSR II (BD).

Cell stimulation and immunoprecipitation. $2 \times 10^{6} \mathrm{~B}$ cells were resuspended in plain Iscove's medium and stimulated with $20 \mathrm{ng} / \mathrm{ml} \mathrm{NIP-BSA}$ at $37^{\circ} \mathrm{C}$ for the times indicated in the figures. Pellets were lysed on ice for 30 min in lysis buffer containing $20 \mathrm{mM}$ Tris- $\mathrm{HCl}$, pH 7.6, $137 \mathrm{mM} \mathrm{NaCl}, 10 \%$ glycerol, $2 \mathrm{mM}$ EDTA, $500 \mu \mathrm{M}$ sodium orthovanadate, $1 \mathrm{mM} \mathrm{NaF}, 0.5 \%$ Brij96, and protease inhibitor cocktail (Sigma-Aldrich). Where indicated in the figures, immunoprecipitation with anti-flag antibody was performed. Lysates were separated by SDS-PAGE, and Western blot analysis was made using an enhanced chemiluminescence system (Thermo Fisher Scientific).

Stably transfected HEK293 cells were lysed on ice for $30 \mathrm{~min}$ in Ex-250 lysis buffer (20 mM Hepes, pH 7.5, $250 \mathrm{mM} \mathrm{NaCl}, 0.5 \mathrm{mM} \mathrm{MgCl}_{2}, 0.5 \%$ $\mathrm{NP}-40$ ). The lysates were centrifuged for $10 \mathrm{~min}$ at $1,000 \mathrm{~g}$, and the supernatant was further diluted with Ex-0 $(20 \mathrm{mM}$ Hepes, $\mathrm{pH} 7.5,0.5 \mathrm{mM}$ $\mathrm{MgCl}_{2}, 0.5 \% \mathrm{NP}-40$ ) to a final concentration of $150 \mathrm{mM} \mathrm{NaCl}$ and centrifuged for $20 \mathrm{~min}$ at $20,000 \mathrm{~g}$. Lysates were immunoprecipitated with antiHA antibody (12CA5) and $15 \mu \mathrm{l}$ of protein G sepharose (50\% slurry). The beads were washed with Ex-150 buffer $(20 \mathrm{mM}$ Hepes, $\mathrm{pH}$ 7.5, $150 \mathrm{mM}$ $\left.\mathrm{NaCl}, 0.5 \mathrm{mM} \mathrm{MgCl}_{2}, 0.5 \% \mathrm{NP}-40\right)$ and PBS, and were finally resuspended in $50 \mu \mathrm{l} \mathrm{PBS}$.

FACS analysis. Cells were resuspended in PBS, $1 \% \mathrm{FCS}$, and $0.01 \% \mathrm{NaN}_{3}$ and were incubated on ice with anti- $\kappa$-biotin and anti- $\lambda$-biotin (SouthernBiotech) followed by streptavidin-Cy5 (Jackson ImmunoResearch Laboratories, Inc.) or with anti-flag-APC (PerkinElmer) and goat anti-mouse
IgM-Alexa Fluor 647 (Invitrogen). Dead cells were excluded by staining with $10 \mu \mathrm{g} / \mathrm{ml}$ propidium iodide. FACS analysis was performed with a flow cytometer (FACSCalibur; BD).

Online supplemental material. Fig. S1 shows the specificity of the anti$\mathrm{R}^{\mathrm{m}}$-Ig $\alpha$ antibody. Fig. S2 depicts BCR and pre-BCR expression in WT or mutant Ig $\alpha$-expressing cells. Fig. S3 shows PRMT1 expression in B cells. Fig. S4 shows the statistical analysis of the in vitro differentiation assay. Online supplemental material is available at http://www.jem.org/cgi/content/ full/jem.20091303/DC1.

The authors would like to dedicate this work to the memory of the late Dr. Koshland Jr., whose work on the methylation of chemotaxis receptors and signaling in bacteria had an early and important impact on the emerging field of signaling.

We thank Dr. E. Hobeika for generating Ig $\alpha$-deficient pro-B cells; H. Schley,

R. Vogt, U. Stauffer, and U. Zeissler for technical assistance; S. Hobitz and A. Wuerch for cell sorting; Dr. P.J. Nielsen for critical reading of the manuscript; and Drs. S. Saccani and D. Van Essen for helpful discussion.

This work was supported by the Swiss National Foundation (fellowship PBBEB-112763), the Human Frontier Science Program career development award, the European Research Council, the Epigenome Network of Excellence (to R. Schneider), the Deutsche Forschungsgemeinschaft (through SFB746), and the Excellence Initiative of the German federal and state governments (EXC294).

The authors have no conflicting financial interests.

Submitted: 15 June 2009

Accepted: 11 February 2010

\section{REFERENCES}

Amin, R.H., and M.S. Schlissel. 2008. Foxo1 directly regulates the transcription of recombination-activating genes during B cell development. Nat. Immunol. 9:613-622. doi:10.1038/ni.1612

Bedford, M.T. 2007. Arginine methylation at a glance. J. Cell Sci. 120:42434246. doi:10.1242/jcs.019885

Bedford, M.T., and S.G. Clarke. 2009. Protein arginine methylation in mammals: who, what, and why. Mol. Cell. 33:1-13. doi:10.1016/j.molcel.2008.12.013

Blanchet, F., A. Cardona, F.A. Letimier, M.S. Hershfield, and O. Acuto. 2005. CD28 costimulatory signal induces protein arginine methylation in T cells. J. Exp. Med. 202:371-377. doi:10.1084/jem.20050176

Blanchet, F., B.T. Schurter, and O. Acuto. 2006. Protein arginine methylation in lymphocyte signaling. Curr. Opin. Immunol. 18:321-328. doi:10.1016/j.coi.2006.03.001

Colucci, F., C. Soudais, E. Rosmaraki, L. Vanes, V.L. Tybulewicz, and J.P. Di Santo. 1999. Dissecting NK cell development using a novel alymphoid mouse model: investigating the role of the c-abl proto-oncogene in murine NK cell differentiation. J. Immunol. 162:2761-2765.

Flemming, A., T. Brummer, M. Reth, and H. Jumaa. 2003. The adaptor protein SLP-65 acts as a tumor suppressor that limits pre-B cell expansion. Nat. Immunol. 4:38-43. doi:10.1038/ni862

Gary, J.D., and S. Clarke. 1998. RNA and protein interactions modulated by protein arginine methylation. Prog. Nucleic Acid Res. Mol. Biol. 61:65-131. doi:10.1016/S0079-6603(08)60825-9

Gay, D., T. Saunders, S. Camper, and M. Weigert. 1993. Receptor editing: an approach by autoreactive B cells to escape tolerance. J. Exp. Med. 177:999-1008. doi:10.1084/jem.177.4.999

Gazumyan, A., A. Reichlin, and M.C. Nussenzweig. 2006. Ig $\beta$ tyrosine residues contribute to the control of $\mathrm{B}$ cell receptor signaling by regulating receptor internalization. J. Exp. Med. 203:1785-1794. doi:10.1084/jem.20060221

Grucza, R.A., K. Fütterer, A.C. Chan, and G. Waksman. 1999. Thermodynamic study of the binding of the tandem-SH2 domain of the Syk kinase to a dually phosphorylated ITAM peptide: evidence for two conformers. Biochemistry. 38:5024-5033. doi:10.1021/bi9829938

Herzog, S., and H. Jumaa. 2007. The $\mathrm{N}$ terminus of the non-T cell activation linker (NTAL) confers inhibitory effects on pre-B cell differentiation. J. Immunol. 178:2336-2343.

Herzog, S., E. Hug, S. Meixlsperger, J.H. Paik, R.A. DePinho, M. Reth, and H. Jumaa. 2008. SLP-65 regulates immunoglobulin light chain gene 
recombination through the PI(3)K-PKB-Foxo pathway. Nat. Immunol. 9:623-631. doi:10.1038/ni.1616

Herzog, S., M. Reth, and H. Jumaa. 2009. Regulation of B-cell proliferation and differentiation by pre-B-cell receptor signalling. Nat. Rev. Immunol. 9:195-205.

Kraus, M., M.B. Alimzhanov, N. Rajewsky, and K. Rajewsky. 2004. Survival of resting mature $\mathrm{B}$ lymphocytes depends on BCR signaling via the Igalpha/ beta heterodimer. Cell. 117:787-800. doi:10.1016/j.cell.2004.05.014

Kulathu, Y., E. Hobeika, G. Turchinovich, and M. Reth. 2008. The kinase Syk as an adaptor controlling sustained calcium signalling and B-cell development. EMBO J. 27:1333-1344. doi:10.1038/emboj.2008.62

Lawson, B.R., Y. Manenkova, J. Ahamed, X. Chen, J.P. Zou, R. Baccala, A.N. Theofilopoulos, and C. Yuan. 2007. Inhibition of transmethylation down-regulates CD4 T cell activation and curtails development of autoimmunity in a model system. J. Immunol. 178:5366-5374.

Matthias, P., and A.G. Rolink. 2005. Transcriptional networks in developing and mature B cells. Nat. Rev. Immunol. 5:497-508.

Meixlsperger, S., F. Köhler, T. Wossning, M. Reppel, M. Müschen, and H. Jumaa. 2007. Conventional light chains inhibit the autonomous signaling capacity of the B cell receptor. Immunity. 26:323-333. doi:10.1016/j.immuni.2007.01.012

Mowen, K.A., B.T. Schurter, J.W. Fathman, M. David, and L.H. Glimcher. 2004. Arginine methylation of NIP45 modulates cytokine gene expression in effector T lymphocytes. Mol. Cell. 15:559-571. doi:10.1016/j.molcel.2004.06.042

Najbauer, J., B.A. Johnson, A.L. Young, and D.W. Aswad. 1993. Peptides with sequences similar to glycine, arginine-rich motifs in proteins interacting with RNA are efficiently recognized by methyltransferase(s) modifying arginine in numerous proteins. J. Biol. Chem. 268:10501-10509.

Nemazee, D.A., and K. Bürki. 1989. Clonal deletion of B lymphocytes in a transgenic mouse bearing anti-MHC class I antibody genes. Nature. 337:562-566. doi:10.1038/337562a0
Pelanda, R., E. Hobeika, T. Kurokawa, Y. Zhang, S. Kuppig, and M. Reth. 2002. Cre recombinase-controlled expression of the mb-1 allele. Genesis. 32:154-157. doi:10.1002/gene.10070

Reth, M. 1989. Antigen receptor tail clue. Nature. 338:383-384. doi:10.1038/338383b0

Reth, M., G.J. Hämmerling, and K. Rajewsky. 1978. Analysis of the repertoire of anti-NP antibodies in C57BL/6 mice by cell fusion. I. Characterization of antibody families in the primary and hyperimmune response. Eur. J. Immunol. 8:393-400. doi:10.1002/eji.1830080605

Rolink, A., A. Kudo, H. Karasuyama, Y. Kikuchi, and F. Melchers. 1991. Long-term proliferating early pre B cell lines and clones with the potential to develop to surface Ig-positive, mitogen reactive $\mathrm{B}$ cells in vitro and in vivo. EMBO J. 10:327-336.

Rolli, V., M. Gallwitz, T. Wossning, A. Flemming, W.W. Schamel, C. Zürn, and M. Reth. 2002. Amplification of B cell antigen receptor signaling by a Syk/ITAM positive feedback loop. Mol. Cell. 10:10571069. doi:10.1016/S1097-2765(02)00739-6

Scharenberg, A.M., L.A. Humphries, and D.J. Rawlings. 2007. Calcium signalling and cell-fate choice in B cells. Nat. Rev. Immunol. 7:778-789.

Spannhoff, A., R. Heinke, I. Bauer, P. Trojer, E. Metzger, R. Gust, R. Schüle, G. Brosch, W. Sippl, and M. Jung. 2007. Target-based approach to inhibitors of histone arginine methyltransferases. J. Med. Chem. 50:2319-2325. doi:10.1021/jm061250e

Srinivasan, L., Y. Sasaki, D.P. Calado, B. Zhang, J.H. Paik, R.A. DePinho, J.L. Kutok, J.F. Kearney, K.L. Otipoby, and K. Rajewsky. 2009. PI3 kinase signals BCR-dependent mature B cell survival. Cell. 139:573-586. doi:10.1016/j.cell.2009.08.041

Yamagata, K., H. Daitoku, Y. Takahashi, K. Namiki, K. Hisatake, K. Kako, H. Mukai, Y. Kasuya, and A. Fukamizu. 2008. Arginine methylation of FOXO transcription factors inhibits their phosphorylation by Akt. Mol. Cell. 32:221-231. doi:10.1016/j.molcel.2008.09.013 


\section{University Library}

\section{- M M N E R VA A gateway to Melbourne's research publications}

Minerva Access is the Institutional Repository of The University of Melbourne

Author/s:

Infantino, S;Benz, B;Waldmann, T;Jung, M;Schneider, R;Reth, M

Title:

Arginine methylation of the B cell antigen receptor promotes differentiation.

Date:

2010-04-12

Citation:

Infantino, S., Benz, B., Waldmann, T., Jung, M., Schneider, R. \& Reth, M. (2010). Arginine methylation of the B cell antigen receptor promotes differentiation.. J Exp Med, 207 (4), pp.711-719. https://doi.org/10.1084/jem.20091303.

Persistent Link:

http://hdl.handle.net/11343/257212

License:

CC BY-NC-SA 